\title{
Cryptanalysis and Further Improvement of a Certificate less Aggregate Signature Scheme
}

\author{
Pankaj Kumar ${ }^{1}$, Vishnu Sharma ${ }^{2}$, Vinod Kumar ${ }^{3}$, Ankush Kumar ${ }^{4}$ \\ ${ }^{1,2}$ School of Computing Science and Engineering, Galgotias University, India \\ ${ }^{3}$ Department of Mathematics, P.G.D.A.V. College, University of Delhi, New Delhi, India \\ ${ }^{4}$ Department of Mathematics, Shivaji College, University of Delhi, New Delhi, India
}

\begin{abstract}
Certificateless aggregate signature reduces nsignatures on $n$ distinct messages from $n$ distinct users into a compact single length signature. Recently Deng et al proposed CLAS Scheme which is an improvement of Hou et al scheme and claims that their scheme is secure against type I type II adversary but unfortunately it is found insecure by against the "Honest but Curious" attack by adversary II. In this paper, we demonstrate that Deng et al proposed CLAS scheme is insecure against type II adversary and suggest an improved CLAS scheme. Keywords: Keywords: Digital Signature, Cryptography, Cryptanalysis, Security attacks.
\end{abstract}

\section{Introduction}

Aggregate signature scheme is helpful for the real time application such as limited bandwidth and low computation. Boneh et al [1] proposed the concept of aggregate signature scheme in 2003. Certificate signature allows mapping nsignatures on $n$ distinct messages from $n$ distinct users into a single length signature. Al-riyami and Paterson [2] proposed the concept of certificateless public key cryptography (CL-PKC) in 2003 which theyprovide the solution of key escrow problem that inherit from the Identity based public key cryptography. In CL-PKC, third party called Key Generation Center (KGC) involves for generating the user's partial private key and user select their private key by using the secret value. Result of this activity escape with the key escrow problem because user secret key is not completely known by the Private Key Generator (PKG) as in Identity based cryptography. Identity based public key cryptography scheme was introduced by Shamir et al [3] in 1984 which gives the solution of key authentication of sender but creates the well-known problem key escrow problem. In identity based cryptography third party Private Key Generator (PKG) generate the whole private key of the user while user select his public by any identity such as email or license number, address number etc that create the key escrow problem. According to the Al-riyami and Paterson [2], CLAS scheme have two types of adversary called type I and type II. Type I adversary has potential to replace the public key of user while have no control on the master key of the user. Type II adversary knows the master key of the KGC while it cannot replace the public key of the user. Furthermore, Huang et al [5] classify these adversaries on their potential power such as Super type, Strong type, Normal type adversary I and adversary II and proposed two CLAS scheme in which first scheme is secure against Normal type I and Super type II where second scheme is secure against Super type I and type II. Liu et al [6] proposed an CLAS scheme in which they proves their scheme is unforgeable against adaptive chosen message attack but Zheng and Wang [7] found insecure Liu et al [6] CLAS scheme by applying concrete attacks with type II adversary. Xiong et al [8] proposed an certificateless aggregate signature scheme and that their CLAS scheme is secure against adaptive chosen message attack of type I and Type II adversary but Hou et al [10] found their scheme is insecure and gives an improvement CLAS scheme. Cheng et al [9] also proves that Xiong et al [8] scheme is also insecure against honest but curious and malicious but passive attack. Deng et al [4] proves that Hou et al [10] scheme is insecure against malicious but passive attack and gives an improvement CLAS scheme and show that this scheme is secure against malicious but passive attack. In this paper we prove that Deng et al [4] is insecure against the honest but curious attack by type II adversary while it is secure against malicious but passive attack.

Paper organization:In Section 2, we presents a review of Deng et al [4] CLS scheme and CLAS scheme and apply an attack honest but curious on Deng et al aggregate with same attack on Deng et al [4] aggregate signature scheme in section 3. Section 4 suggests a modified CLAS scheme to improve Deng et al [4]. Finally, the conclusions are presents in Section 5. 


\section{Review of the Deng et al [4] CLS Scheme}

The symbol table is given below.

TABLE 1 Symbol used in scheme

\begin{tabular}{|c|l|}
\hline Symbols & Description \\
\hline$s$ & The master key of KGC \\
\hline$P$ & Generator of the group \\
\hline$n, q$ & Natural number \\
\hline$P_{p u b}$ & The public key of KGC \\
\hline$I D_{i}$ & The User's identity \\
\hline$u s k_{I D_{i}}$ & The Users secret key \\
\hline$p s k_{I D_{i}}$ & The partial private key of identity $I D_{i}$ \\
\hline$P a r a m s$ & The system parameters generated by KGC \\
\hline$\left(u s k_{I D_{i}}, u p k_{I D_{i}}\right)$ & The user's secret / public key pair of identity $I D_{i}$ \\
\hline$u p k_{I D_{i}}$ & The public key of identity $I D_{i}$ \\
\hline$m_{i}$ & The message corresponding to user's identity $I D_{i}$ \\
\hline$\sigma_{i}$ & Signature on the message $m_{i}$ with user's identity $I D_{i}$ \\
\hline$V$ & Signature of user corresponding to user's $I D_{i}$ \\
\hline KGC & Key Generation Center \\
\hline CLS & Certificateless Signature \\
\hline CLAS & Certificateless Aggregate Signature \\
\hline &
\end{tabular}

In this section we give a brief review of Deng et al CLAS scheme. Deng et al CLS scheme consist of five algorithms Masterkeygen, Partialkeygen, Userkeygen, Sign, Verify.

Masterkeygen: on taking a security input $k, \mathrm{KGC}$ starts the algorithm as follow:

i) Generate two groups one is cyclic additive group $G_{1}$ and second is cyclic multiplicative group $G_{2}$ having the same order $q$ with two generator $P, Q$ of $G_{1}$ and a bilinear pairing $e: G_{1} \times G_{2} \rightarrow G_{T}$

ii) Select a random number $s \in Z_{q}^{*}$ and computes $P_{p u b}=s P$, taking $s$ as a master key of KGC and $P_{p u b}$ as a public key of KGC.

iii) Select four hash functions $H_{1}:\{0,1\}^{*} \rightarrow G_{1}, H_{2}:\{0,1\}^{*} \rightarrow G_{1}, H_{3}:\{0,1\}^{*} \rightarrow Z_{q}^{*}, H_{4}:\{0,1\}^{*} \rightarrow Z_{q}^{*}$.

iv) Generates the system parameters say Params are $\left\{q, G_{1}, G_{2}, e, P, Q, P_{p u b}, H_{1}, H_{2}, H_{3}, H_{4}\right\}$ and keep secretly master key $s$ by KGC.

Partialkeygen: After taking input user's identity $I D_{i}$, The KGC first computes the user's partial private key $p s k_{I D_{i}}=s Q_{I D_{i}}$ where $Q_{I D_{i}}=H_{1}\left(I D_{i}\right)$ and forward it to the user via a secure way.

Userkeygen: The user chooses a random number $x_{I D_{i}} \in Z_{q}^{*}$ and set as secret key $u s k_{I D i}$, then computes its public key $u p k_{I D_{i}}=u s k_{I D_{i}} \cdot P$

Sign: The user with identity $I D_{i}$ takes the Params, the partial private key $p s k_{I D_{i}}$, corresponding secret key $u s k_{I D i}$ and then performs the following steps to generate the signature:

i) Select a random number $r_{i} \in Z_{q}^{*}$ and computes

$U_{i}=r_{i} . P, h_{1 i}=H_{3}\left(m_{i}, I D_{i}, u p k_{i D_{i}}, U_{i}\right), h_{2 i}=H_{4}\left(m_{i}, I D_{i}, u p k_{i D_{i}}, U_{i}\right), K=H_{2}\left(q, P, P_{p u b}\right)$

ii) Compute: $V_{i}=p s k_{I D_{i}}+h_{1 i} r_{i} P+h_{2 i} x_{i} K$

iii) Provides a signature $\left(U_{i}, V_{i}\right)$ on message $m_{i}$.

Verify: Given a signature $\left(U_{i}, V_{i}\right)$ with message $m_{i}$ corresponding public key $u p k_{I D_{i}}$ regarding the identity $I D_{i}$ verifier performs the following steps:

i) Computes $U_{i}=r_{i} . P, h_{1 i}=H_{3}\left(m_{i}, I D_{i}, u p k_{i D_{i}}, U_{i}\right), h_{2 i}=H_{4}\left(m_{i}, I D_{i}, u p k_{i D_{i}}, U_{i}\right), K=H_{2}\left(q, P, P_{p u b}\right)$

ii) Verify the following equation

$e\left(V_{i}, P\right)=e\left(Q_{I D_{i}}+h_{1 i} U_{i}, P_{p u b}\right) e\left(h_{2 i} u p k_{I D_{i}}, K\right)$

If it satisfied then accept the signature. 


\subsection{Deng et al [4] CLAS scheme}

CLAS scheme consist of seven steps in which five algorithms Masterkeygen, Partialkeygen, Userkeygen, Sign, Verify are same as CLS scheme and two extra algorithms say Aggregate and Aggregate verify are involved in CLAS scheme whose description is given below:

1) Aggregate: for an aggregating set of $n$ users $\left\{U_{1}, U_{2} \ldots \ldots \ldots, U_{n}\right\}$ with their identities $\left\{I D_{1}, I D_{2} \ldots \ldots \ldots, I D_{n}\right\}$ and the corresponding public keys $\left\{u p k_{1}, u p k_{2}, \ldots \ldots \ldots, u p k_{n}\right\}$, and with signature pairs $\left\{\left(m_{1}, \sigma_{1}=\left(U_{1}, V_{1}\right)\right), \ldots \ldots \ldots\left(m_{n}, \sigma_{n}=\left(U_{n}, V_{n}\right)\right\}\right.$, then aggregator computes $V=\sum_{i=1}^{n} V_{i}$ and results an aggregate signature as $\sigma=\left(U_{1}, U_{2} \ldots \ldots . ., U_{n}, V\right)$.

2) Aggregate Verify: for verify an aggregate signature $\sigma=\left(U_{1}, U_{2} \ldots \ldots \ldots, U_{n}, V\right)$ signing by $n$ users $\left\{U_{1}, U_{2} \ldots \ldots \ldots ., U_{n}\right\}$ with their identities $\left\{I D_{1}, I D_{2} \ldots \ldots \ldots, I D_{n}\right\}$, verifier performs the following steps:

i) Computes $\quad Q_{I D_{i}}=H_{1}\left(I D_{i}\right), \quad h_{1 i}=H_{3}\left(m_{i}, I D_{i}, u p k_{i D_{i}}, U_{i}\right), \quad h_{2 i}=H_{4}\left(m_{i}, I D_{i}, u p k_{i D_{i}}, U_{i}\right)$, $K=H_{2}\left(q, P, P_{p u b}\right)$

ii) Verify

$$
e(V, P)=e\left(\sum_{i=1}^{n}\left(h_{1 i} \cdot U_{i}+Q_{I D_{i}}\right), P_{p u b}\right) \cdot e\left(\sum h_{2 i} \cdot u p k_{I D_{i}}, K\right)
$$

\section{Cryptanalysis of Deng et al [4] CLS scheme}

In this subsection we discuss the type II attack on behalf we claim that proposed CLS scheme is insecure. Since KGC knows the master key, then KGC compute the value $r_{i} P_{p u b}=r_{i} s=s r_{i} P=s U_{i}$. Since $s$ and $U$ are publicly known and with the help of known value $s U_{i}$ we calculate $r_{i} P_{p u b}$. With the help of master key KGC can compute the partial private key of user $p s k_{I D_{i}}$, by $p s k_{I D_{i}}=s Q_{I D_{i}}$ while $Q_{I D_{i}}=H_{1}\left(I D_{i}\right)$ is known quantity. $h_{1 i}$ and $h_{2 i}$ are the hash value then $h_{2 i}^{-1}$ also compute. Now he can compute the fix value $x_{i} K=h_{2 i}^{-1}\left(V^{*}-p s k_{I D_{i}}-h_{1 i} s U_{i}^{*}\right) \quad$ by capturing the signature $\left(U^{*}, V^{*}\right)$ onmessage $m_{i}$, $h_{1 i}=H_{3}\left(m_{i}^{*}, I D_{i}, u p k_{i D_{i}}, U_{i}^{*}\right) h_{2 i}=H_{4}\left(m_{i}^{*}, I D_{i}, u p k_{i D_{i}}, U_{i}^{*}\right)$. Since KGC don't know the user's secret key but he knowns about the fix value $x_{i} K$ then he can forge user's signature on any message in aggregate set. The description of this attack is given below:

Intercept partial signature: in the first step KGC intercept the signature of user $U_{i}$ with the identity $I D_{i}$ corresponding public key $u p k_{I D_{i}}$ and find the signature $\left(U^{*}, V^{*}\right)$.

\section{Compute fix value:}

i) Compute $h_{1 i}=H_{3}\left(m_{i}^{*}, I D_{i}, u p k_{i D_{i}}, U_{i}^{*}\right) h_{2 i}=H_{4}\left(m_{i}^{*}, I D_{i}, u p k_{i D_{i}}, U_{i}^{*}\right), K=H_{2}\left(q, P, P_{p u b}\right)$

ii) Compute $r_{i}^{*} P_{p u b}=r_{i}^{*} s P=s r_{i} P=s U_{i}^{*}$

iii) Computes $x_{i} K=h_{2 i}^{-1}\left(V^{*}-p s k_{I D_{i}}-h_{1 i} s U_{i}^{*}\right)$

Forge partial signature: Now KGC perform the following step to forge CLS signature $\left(U_{i}^{\prime}, V_{i}^{\prime}\right)$ on message $m_{i}^{\prime}$.

i) Select $U_{i}^{\prime} \in G_{1}$, and extract the value of $s U_{i}$ from $r_{i} P_{p u b}$.

ii) Computes $h_{1 i}=H_{3}\left(m_{i}^{\prime}, I D_{i}, u p k_{i D_{i}}, U^{\prime}\right) h_{1 i}=H_{4}\left(m_{i}^{\prime}, I D_{i}, u p k_{i D_{i}}, U_{i}^{\prime}\right), K=H_{2}\left(q, P, P_{p u b}\right)$

iii) Computes $V_{i}^{\prime}=p s k_{I D_{i}}+h_{1 i} s U_{i}^{\prime}+h_{2 i} x_{i} K$

Then he provides an output $\left(U_{i}^{\prime}, V_{i}^{\prime}\right)$ on the message $m_{i}^{\prime}$.

\section{Verification:}

$$
\begin{aligned}
e\left(V_{i}^{\prime}, P\right) & =e\left(p s k_{I D_{i}}+h_{1 i} s U_{i}^{\prime}+h_{2 i} x_{i} K, P\right) \\
& =e\left(p s k_{I D_{i}}+h_{1 i} s U_{i}^{\prime}, P\right) e\left(h_{2 i} x_{i} K, P\right)
\end{aligned}
$$




$$
\begin{aligned}
& =e\left(s Q_{I D_{i}}+h_{1 i} s U_{i}^{\prime}, P\right) e\left(h_{2 i} x_{i} P, K\right) \\
& =e\left(Q_{I D_{i}}+h_{1 i} U_{i}^{\prime}, s P\right) e\left(h_{2 i} u p k_{I D_{i}}, K\right) \\
& =e\left(Q_{I D_{i}}+h_{1 i} U_{i}^{\prime}, P_{p u b}\right) e\left(h_{2 i} u p k_{I D_{i}}, K\right)
\end{aligned}
$$

\subsection{Cryptanalysis of Deng [4] CLAS scheme}

KGC can compute $r_{i} P_{p u b}$ and $x_{i} K$ of any user's signature by the above method mention used to forge the CLS scheme. Then he can club the entire signatures to forge the aggregate signature.

Now KGC calculates $V^{* *}=\sum_{i=1}^{n} V^{\prime}$ and provide the output $\left(U_{1}^{\prime}, U_{2}^{\prime} \ldots \ldots \ldots . . ., U_{n}^{\prime}, V^{* *}\right)$ as the forge aggregate signature.

For $i \in[1, n], Q_{i}=H_{1}\left(I D_{i}\right), h_{1 i}=H_{3}\left(m_{i}^{\prime}, I D_{i}, u p k_{i D_{i}}, U_{i}^{\prime}\right), h_{1 i}=H_{4}\left(m_{i}^{\prime}, I D_{i}, u p k_{i D_{i}}, U_{i}^{\prime}\right), K=H_{2}\left(q, P, P_{p u b}\right)$

Forge aggregate signature is valid if it satisfied the following equation.

$$
e\left(V^{* *}, P\right)=e\left(\sum_{i=1}^{n}\left(h_{1 i} \cdot U_{i}^{\prime}+Q_{I D_{i}}\right), P_{p u b}\right) e\left(\sum h_{2 i} \cdot u p k_{I D_{i}}, K\right)
$$

\section{Improved Certificateless Signature Scheme}

We propose a modified CLAS scheme to remove the weakness of Deng et al CLAS scheme.

Masterkeygen: on taking a security input $k, \mathrm{KGC}$ starts the algorithm as follow:

i) Generate two groups one is cyclic additive group $G_{1}$ and second is cyclic multiplicative group $G_{2}$ having the same order $q$ with two generator $P, Q$ of $G_{1}$ and a bilinear pairing $e: G_{1} \times G_{2} \rightarrow G_{T}$

ii) Select a random number $s \in Z_{q}^{*}$ and computes $P_{p u b}=s P$, taking $s$ as a master key of KGC and $P_{p u b}$ as a public key of KGC.

iii) Select four hash functions $H_{1}:\{0,1\}^{*} \rightarrow G_{1}, H_{2}:\{0,1\}^{*} \rightarrow G_{1}, H_{3}:\{0,1\}^{*} \rightarrow Z_{q}^{*}, H_{4}:\{0,1\}^{*} \rightarrow Z_{q}^{*}$.

iv) Generates the system parameters say Params are $\left\{q, G_{1}, G_{2}, e, P, Q, P_{p u b}, H_{1}, H_{2}, H_{3}, H_{4}\right\}$ and keep secretly master key $s$ by KGC.

Partialkeygen: After taking input user's identity $I D_{i}$, The KGC first computes the user's partial private key $p s k_{I D_{i}}=s Q_{I D_{i}}$ where $Q_{I D_{i}}=H_{1}\left(I D_{i}\right)$ and forward it to the user via a secure way.

Userkeygen: The user chooses a random number $x_{I D_{i}} \in Z_{q}^{*}$ and set as secret key $u s k_{I D_{i}}$, then computes its public key $u p k_{I D_{i}}=u s k_{I D_{i}} \cdot P$

Sign: The user with identity $I D_{i}$ takes the Params, the partial private key $p s k_{I D_{i}}$, corresponding secret key $u s k_{I D i}$ and then performs the following steps to generate the signature:

iv) Select a random number $r_{i} \in Z_{q}^{*}$ and computes

$U_{i}=r_{i} . P, \quad h_{1 i}=H_{3}\left(m_{i}, I D_{i}, u p k_{i D_{i}}, U_{i}\right), \quad h_{2 i}=H_{4}\left(m_{i}, I D_{i}, u p k_{i D_{i}}, U_{i}\right), \quad K=H_{2}\left(q, P, P_{p u b}\right)$, $T=H_{2}\left(q, P, P_{p u b}\right)$

v) Compute: $V_{i}=p s k_{I D_{i}}+h_{1 i} r_{i} T+h_{2 i} x_{i} K$

vi) Provides a signature $\left(U_{i}, V_{i}\right)$ on message $m_{i}$.

Verify: Given a signature $\left(U_{i}, V_{i}\right)$ with message $m_{i}$ corresponding public key $u p k_{I D_{i}}$ regarding the identity $I D_{i}$ verifier performs the following steps:

iii) Computes $U_{i}=r_{i} . P, h_{1 i}=H_{3}\left(m_{i}, I D_{i}, u p k_{i D_{i}}, U_{i}\right), h_{2 i}=H_{4}\left(m_{i}, I D_{i}, u p k_{i D_{i}}, U_{i}\right), K=H_{2}\left(q, P, P_{p u b}\right)$

iv) Verify the following equation

$$
e\left(V_{i}, P\right)=e\left(Q_{I D_{i}}+h_{1 i} U_{i}, P_{p u b}\right) e\left(h_{2 i} u p k_{I D_{i}}, K\right)
$$




\section{Conclusion}

Recently, Deng et al [4] proposed an efficient improvedCLAS scheme of Hou et al [10] CLAS scheme. In this paper, we first give a detail review of Deng et al CLS and CLAS scheme then show that proposed CLAS is insecure against concrete attacks. We point out that the security leaks of the scheme that is depends on the user secret key $u s k_{I D_{i}}$ and a random number $r_{i}$ select by the user but malicious KGC computes the fixed value $r_{i} P_{p u b}, x_{i} K$ and forge the signature without the help of $u s k_{I D_{i}}$ and $r_{i}$. We proposed a certificateless signature scheme to remove the security leaks arise in Deng et al [4].

\section{References}

[1]. D. Boneh, C. Gentry, B. Lynn, H. Shacham, “Aggregate and Verifiably Encrypted Signatures from Bilinear Maps”, E. Biham (Ed.), EUROCRYPT 2003, LNCS 2656, Springer-Verlag, Warsaw, Poland, 2003, pp. 416-432.

[2]. Al-Riyami, S., Paterson, K. “Certificateless Public Key Cryptography”, Asiacrypt' 03, LNCS 2894, Springer-Verlag. (2003) pp. $452-473$.

[3]. A. Shamir, "Identity Based Cryptosystems and Signature Schemes",G.R. Blakley, D. Chaum (Eds.), Crypto'84, LNCS 196, Springer-Verlag, Santa Barbara, California, USA, 1984, pp. 47-53.

[4]. J. Deng, C. Xu, H. Wu, G. Yang, “An Improved Certificateless Aggregate Signature", 2014 IEEE Internatational Conference on Computer and Information Technology pp 919-922.

[5]. X. Huang, Y. Mu, W. Susilo, D. S. Wong, W. Wu, "Certificateless Signatures: New Scheme and Security Models" The Computer Journal, Vol. 55 No.4, 2012 pp 457-474.

[6]. H Liu, S Wang, M Liang and Y Chen, "New Construction of Efficient Certificateless Aggregate Signatures", International Journal of Security and Its Applications Vol.8, No.1 (2014), pp. 411-422.

[7]. Y. Zhang, C. Wang “ Comment on New Construction of Efficient Certificateless, Aggregate Signatures" International Journal of Security and Its Applications Vol.9, No.1 (2015), pp.147-154.

[8]. H. Xiong, Z. Guan, Z. Chen, F. Li "An Efficient Certificateless Aggregate Signature With Constant Pairing Computations" Inform. Sci. 219 (2013) 225-235.

[9]. Lin Cheng, Qiaoyan Wen, Zhengping Jin, Hua Zhang, Liming Zhou" Cryptanalysis and Improvement of a Certificateless Aggregate Signature Scheme " information sciences 295 (2015) pp 337-46

[10]. H.Hou, X.Zhang,X.Dong, "Improved Certificateless Aggregate Signature Scheme ”, Journal of Shandong University (Natural Science), 48(9),pp. 29-34,2013. 